\title{
Aarnoo Simultaneidade de doenças crônicas não transmissíveis em 2013 nas capitais brasileiras: prevalência e perfil sociodemográfico*
}

doi: $10.5123 / S 1679-49742020000100006$

\section{Simultaneity of chronic noncommunicable diseases in 2013 in Brazilian state capital cities: prevalence and demographic profile}

\author{
Simultaneidad de enfermedades crónicas no transmisibles en 2013 en las capitales \\ brasileñas: prevalencia y perfil demográfico
}

\author{
Marina Christofoletti ${ }^{1}$ - $\mathbb{0}$ orcid.org/0000-0002-5672-6869 \\ Giovani Firpo Del Duca' - @ orcid.org/0000-0003-0893-2032 \\ Aline Mendes Gerage ${ }^{1}$ - Dorcid.org/0000-0002-0555-5422 \\ Deborah Carvalho Malta ${ }^{2}$ - (1) orcid.org/0000-0002-8214-5734 \\ 'Universidade Federal de Santa Catarina, Departamento de Educação Física, Florianópolis, SC, Brasil \\ Universidade Federal de Minas Gerais, Departamento de Enfermagem Materno-Infantil e Saúde Pública, Belo Horizonte, MG, Brasil
}

\section{Resumo}

Objetivo: descrever a prevalência e o perfil sociodemográfico da simultaneidade de doenças crônicas não transmissíveis (DCNTs) em adultos e idosos das capitais brasileiras. Métodos: Sistema de Vigilância de Fatores de Risco e Proteção para Doenças Crônicas não Transmissíveis (Vigitel), realizado em 2013; a simultaneidade considerou duas ou mais DCNTs (diabetes mellitus, dislipidemia, hipertensão e obesidade). Resultados: dos 52.929 participantes, $13,7 \%$ dos adultos e $42,9 \%$ dos idosos tiveram simultaneidade de DCNT; houve maior agrupamento da hipertensão com diabetes mellitus em adultos, e hipertensão com obesidade em idosos; a simultaneidade foi mais prevalente em mulheres, na idade entre 50 e 59 anos, com companheiro, e de escolaridade até oito anos de estudo; as cidades com menor e maior prevalência em adultos, respectivamente, foram São Luís e Cuiabá, e em idosos, Belém e Manaus. Conclusão: a simultaneidade foi identificada no contexto nacional; medidas de prevenção devem ser direcionadas especialmente ao tratamento da hipertensão.

Palavras-chave: Doença Crônica; Grupos Diagnósticos Relacionados; Fatores Socioeconômicos; Estudos Transversais.

\footnotetext{
*Artigo derivado da dissertação de mestrado da autora Marina Christofoletti, com o título 'Multimorbidade de doenças crônicas não transmissíveis no Brasil: prevalência e associação com indicadores sociodemográficos, de atividade física e de comportamento sedentário em adultos e idosos', defendido junto à Universidade Federal de Santa Catarina em 2017.
}

Endereço para correspondência:

Marina Christofoletti - Campus Universitário Reitor João David Ferreira Lima, Florianópolis, SC, Brasil. CEP: 88040-230

E-mail:marinachriss@outlook.com 


\section{Introdução}

No Brasil, as doenças crônicas não transmissíveis (DCNTs) foram responsáveis por $73 \%$ das mortes gerais e $17 \%$ das mortes precoces registradas no ano de 2017. ${ }^{1}$ Esses dados, reflexo das elevadas prevalências dessas doenças, atribuem-se à chamada 'Transição Epidemiológica', ${ }^{2}$ fato que pode implicar gastos elevados no sistema público de saúde, aposentadorias precoces e absenteísmo. ${ }^{3}$

As DCNTs, individualmente, promovem uma sobrecarga orgânica nos sistemas corporais acometidos, ${ }^{1-4}$ em função de alterações nos processos fisiológicos, debilitando o estado de saúde e facilitando o aparecimento de outras patologias. ${ }^{4}$ Países como Estados Unidos $^{5}$ e Canadá ${ }^{6}$ retrataram, respectivamente, $26,0 \%$ e $12,9 \%$ da população adulta com dois ou mais diagnósticos de DCNTs no período entre 2014 e 2015. 0 tratamento medicamentoso expõe a população a possíveis efeitos colaterais de interações medicamentosas, ${ }^{7}$ enquanto os impactos coletivos são vinculados aos gastos com serviços de saúde. ${ }^{8}$ A simultaneidade de doenças, conceituada como multimorbidade, consiste na existência do diagnóstico de duas ou mais doenças em co-ocorrência no mesmo indivíduo. ${ }^{8}$ Tal condição preocupa o cenário de Saúde Pública, dado o agravo da iniquidade na saúde e a sobrecarga desses serviços, a exemplo do uso contínuo de medicamentos, atendimentos médicos especializados e hospitalizações decorrentes. ${ }^{9}$

\section{As DCNTs, individualmente, promovem uma sobrecarga orgânica nos sistemas corporais acometidos, em função de alterações nos processos fisiológicos, debilitando o estado de saúde $e$ facilitando o aparecimento de outras patologias}

Ao se considerar o contexto de envelhecimento populacional, revela-se a necessidade de uma resposta abrangente da Saúde Pública na promoção de uma melhor qualidade de vida após os diagnósticos de DCNTs. A simultaneidade de doenças pode ser considerada uma realidade a ser investigada, pois exige maior cuidado com a saúde, especialmente com a sobrevida dos pacientes após os diagnósticos.
A investigação sobre a prevalência da simultaneidade de doenças em estudos populacionais tem sido bastante explorada no contexto brasileiro, principalmente quanto à investigação de fatores associados. ${ }^{10.11}$ Porém, poucos estudos incluem uma ampla faixa etária, particularmente de adultos, bem como o comportamento da agregação das doenças. A presença de diabetes mellitus, dislipidemia, hipertensão e/ou obesidade é considerada importante indicador de risco cardiovascular, segundo o 'Plano de Ações Estratégicas para o Enfrentamento de DCNT', elaborado pelo Ministério da Saúde, ${ }^{12} 0$ que reforça a importância de abordar esses agravos e tratar da saúde como um complexo dinâmico, de entendimento integral, e não exclusivamente como ausência de doenças. Tal entendimento também deve considerar características sociodemográficas, como idade, sexo e indicadores econômicos, capazes de interferir na maior probabilidade de ocorrência e na gravidade dessa simultaneidade. ${ }^{13}$

Com o propósito de contribuir para a compreensão da simultaneidade de DCNTs e os possíveis indicadores desse estado de saúde, o presente estudo teve como objetivo descrever a prevalência e o perfil sociodemográfico da simultaneidade de DCNTs em adultos e idosos residentes nas capitais brasileiras.

\section{Métodos}

0 presente estudo consiste em uma análise de dados obtidos pelo Sistema de Vigilância de Fatores de Risco e Proteção para Doenças Crônicas não Transmissíveis (Vigitel), um inquérito nacional, realizado anualmente desde 2006, em todas as 27 capitais das Unidades da Federação (UFs) brasileira.

A coleta foi realizada de fevereiro a dezembro de 2013, com indivíduos na idade de 18 anos ou mais, residentes em domicílios com linha telefônica fixa. A realização do processo de amostragem considerou os critérios de estimava das variáveis de fatores de risco e proteção para DCNT com nível de confiança de $95 \%$ e erro máximo de 3 pontos percentuais, levando ao tamanho da amostra mínimo de 2.000 entrevistas.

A definição das linhas elegíveis para amostra foi elaborada em três etapas: (i) a seleção estratificada por código de endereçamento postal (CEP), (ii) o reconhecimento das linhas elegíveis, concomitantemente à realização das entrevistas, e (iii) o sorteio 
do participante do inquérito para a realização da entrevista, entre todos os adultos residentes no domicílio. A descrição desse processo, em sua íntegra, encontra-se no relatório publicado pelo Ministério da Saúde..$^{14} 0$ peso pós-estratificação de cada indivíduo da amostra foi calculado pelo método conhecido como 'rake'. ${ }^{14}$ Os dados foram coletados mediante entrevista telefônica e recursos de computador, concomitantemente.

0 instrumento utilizado foi validado em estudos-piloto prévios. ${ }^{14}$ A variável de desfecho foi a simultaneidade de DCNTs. Considerando-se que o sistema Vigitel reúne um limitado número de questões sobre diagnóstico, optou-se por incluir doenças e fatores de risco, como diabetes mellitus, dislipidemia, hipertensão arterial e obesidade, neste estudo denominadas DCNTs. Estas DCNTs foram mensuradas pela resposta afirmativa à pergunta:

Algum médico já lhe disse que o(a) sr(a) tem diabetes/dislipidemia/pressão alta?

Ou considerando-se, para a obesidade, o índice de massa corporal (IMC) acima ou igual a $30 \mathrm{~kg} /$ $\mathrm{m}^{2}$, calculado pelo autorrelato de peso e estatura do entrevistado. Para esta segunda variável, mediante a existência de respostas deixadas em branco $(8,8 \%)$, realizou-se imputação dos dados pela técnica de "bot deck". ${ }^{14}$ A técnica utilizada identificou a ausência da resposta e sua associação com as variáveis 'idade', 'sexo', 'escolaridade' e 'raça/cor da pele'. Posteriormente, foram definidos grupos de acordo com as semelhanças entre características, incluindo aqueles com resposta e sem resposta à questão de peso e/ou estatura. Foi escolhido, aleatoriamente, um participante por capital que tivesse respondido à(s) questão(ões), para ter seus dados replicados no lugar daqueles sem resposta, considerando-se a preexistência de características semelhantes. Mais detalhes metodológicos podem ser vistos em outra publicação. ${ }^{14}$ A variável de simultaneidade foi dicotomizada em ausência (0 ou 1 doença) e presença $(\geq 2$ doenças) de simultaneidade. Analisou-se, ainda, a quantidade de doenças acumuladas (2 doenças, 3 doenças ou 4 doenças).

As variáveis de exposição foram:

- sexo (masculino; feminino);

- idade (em anos: 18 a 29; 30 a 39; 40 a 49; 50 a $59 ; 60$ a $69 ; 70$ a $79 ; 80$ a $89 ; \geq 90)$;
- estado civil (com companheiro; sem companheiro);

- raça/cor da pele (branca; preta/parda);

- escolaridade (em anos de estudo: até 8; entre

9 e 11; igual ou acima de 12);

- macrorregião demográfica (Centro-Oeste; Nordeste; Norte; Sudeste; Sul); e

- as 27 capitais das UFs.

Para descrição, empregaram-se frequências absolutas e relativas, com intervalos de confiança de $95 \%\left(\mathrm{IC}_{95 \%}\right)$. Realizou-se a estratificação dos dados, conforme sexo, idade e relato de simultaneidade de DCNTs. A interpretação dos achados levou em consideração os $\mathrm{IC}_{95 \%}$. Para análise, utilizou-se o software Stata ${ }^{\circledR}$ versão 13.0 (Stata Corporation, College Station, USA). Todas as análises consideraram o peso amostral, ponderado no processo por dois fatores: o inverso do número de linhas telefônicas existentes no domicílio entrevistado e o número de adultos residentes no domicílio do entrevistado.

o Termo de Consentimento Livre e Esclarecido foi registrado por consentimento verbal, haja vista tratar-se de um inquérito telefônico. 0 estudo foi aprovado pela Comissão Nacional de Ética em Pesquisa envolvendo seres humanos, a CONEP, subordinada ao Conselho Nacional de Saúde/ Ministério da Saúde: Parecer nº 355.590.

\section{Resultados}

Entre os 74.005 sujeitos elegíveis, 52.929 entrevistas foram concluídas $(71,5 \%)$. A maior parte da amostra foi representada por mulheres $\left(53,9 \%\right.$ [ $\mathrm{IC}_{95 \%}$ $53,1 ; 54,8])$, pessoas sem companheiro(a) $(50,6 \%$ $\left.\left[\mathrm{IC}_{95 \%} 49,7 ; 21,5\right]\right)$, raça/cor da pele parda/preta $\left(52,7 \%\left[\mathrm{IC}_{95 \%} 51,8 ; 53,6\right]\right)$ e 9 a 11 anos de estudo $\left(37,3 \%\left[\mathrm{IC}_{95 \%} 36,5 ; 38,2\right]\right)$. Quanto às DCNTs, a prevalência de diabetes mellitus foi de $6,9 \%\left(\mathrm{IC}_{95 \%} 6,5 ; 7,3\right)$, $20,3 \%\left(\mathrm{IC}_{95 \%} 19,6 ; 21,0\right)$ referiram dislipidemia, 24,1\% $\left(\mathrm{IC}_{95 \%} 23,4 ; 24,8\right)$ hipertensão arterial e 17,5\% ( IC $_{95 \%}$ $16,9 ; 18,2)$ obesidade.

A quantidade de respostas do desfecho do presente estudo foi de 47.218 (89,2\%). Entre elas, a prevalência de simultaneidade de DCNTs foi de $18,2 \%$ $\left(\mathrm{IC}_{95 \%} 17,5 ; 18,9\right)$. Ao se considerar o escore de doenças acumuladas, constatou-se $12,6 \%\left(\mathrm{IC}_{95 \%} 12,0 ; 13,2\right)$ da amostra com duas, 4,7\%\% ( IC $\left._{95 \%} 4,3 ; 5,1\right)$ com 
três e $0,9 \%\left(\mathrm{IC}_{95 \%}, 0,8 ; 1,1\right)$ com quatro DCNTs. Na estratificação dos resultados conforme a idade, as prevalências de simultaneidade de doenças em adultos e idosos foram, respectivamente, de $13,7 \%$ (IC $_{95 \%}$ $12,7 ; 14,1)$ e $42,9 \%\left(\mathrm{IC}_{95 \%} 41,0 ; 44,9\right)$. As combinações mais frequentes em adultos foram diabetes mellitus $\mathrm{e}$ hipertensão (3,37\%), hipertensão e obesidade (2,9\%) e diabetes mellitus e dislipidemia (2,2\%). Nos idosos, as combinações de duas DCNTs mais apresentadas foram hipertensão e obesidade $(12,2 \%)$ e diabetes mellitus e obesidade (6,3\%); e na simultaneidade de três doenças, 5,6\% com dislipidemia, hipertensão e obesidade, e 4,3\% com diabetes mellitus, hipertensão e obesidade.

A Tabela 1 considera 0 relato de simultaneidade de doenças de acordo com indicadores sociodemográficos. Naqueles sem a simultaneidade de doenças, as estimativas foram semelhantes entre homens e mulheres, com maiores prevalências entre adultos mais jovens (18 a 29 anos $\left[33,7 \%-\mathrm{IC}_{95 \%}\right.$ $32,8 ; 34,8]$ ), pessoas sem companheiro(a) $(52,9 \%$ $\left.\left[\mathrm{IC}_{95 \%} 51,9 ; 53,9\right]\right)$, raça/cor da pele preta ou parda $\left(52,7 \%\left[\mathrm{IC}_{95 \%} 51,6 ; 53,8\right]\right), 9$ a 11 anos de estudo (40,5\% [ $\left.\left.\mathrm{IC}_{95 \%} 39,5 ; 41,5\right]\right)$, e com residência na região Sudeste $\left.\left(44,3 \%\left[\mathrm{IC}_{95 \%} 43,2 ; 45,4\right)\right]\right)$. Na presença de simultaneidade de doenças, a raça/cor da pele não apresentou distinção nas proporções entre grupos. A simultaneidade de DCNTs foi mais prevalente entre as mulheres $\left(56,0 \%\right.$ [IC $\left.\left._{95 \%} 53,8 ; 58,1\right]\right), 50$ e 59 anos de idade $\left(27,2 \%\right.$ [IC $\left.\left._{95 \%} 25,3 ; 29,1\right]\right)$, com companheiros $\left(64,4 \%\right.$ [IC $\left.\left.{ }_{95 \%} 62,4 ; 66,3\right]\right)$, com tempo de estudo de até 8 anos $\left(56,3 \%\right.$ [IC $\left.\left.{ }_{95 \%} 54,3 ; 58,3\right]\right)$, e com residência na região Sudeste $\left(50,6 \%\left[\mathrm{IC}_{95 \%} 48,5 ; 52,6\right]\right)$.

A simultaneidade de DCNTs apresentou variação no que se refere ao agrupamento de doenças conforme a década de vida dos brasileiros (Figura 1). Apresentar duas DCNTs consistiu no agrupamento com maior prevalência a partir dos 30 anos, enquanto três doenças se concentraram principalmente entre os 50 e os 89 anos; a presença simultânea das quatro doenças foi mais evidente na primeira década da faixa etária de idosos (60 a 69 anos). Foi possível identificar que a existência de três e quatro doenças apresenta maiores prevalências entre os 50 e os 59 anos de idade; já as pessoas com idade superior aos 90 anos apresentaram a prevalência de três ou quatro doenças menor, na comparação com as demais faixas etárias de idosos.
A simultaneidade de DCNTs entre adultos nas capitais do Brasil teve um comportamento semelhante. As capitais com menor prevalência de simultaneidade de doenças em adultos foram São Luís (9,7\% [ IC $_{95 \%}$ $8,1 ; 11,7])$, Florianópolis $\left(10,4 \%\left[\mathrm{IC}_{95 \%} 8,7 ; 12,5\right]\right)$ e Porto Velho $\left(11,0 \%\left[\operatorname{IC}_{95 \%} 9,1 ; 13,0\right)\right.$. Por sua vez, Cuiabá $\left(16,8 \%\left[\mathrm{IC}_{95 \%} 14,1 ; 20,0\right]\right)$, Maceió $\left.\left(16,4 \%\left[\mathrm{IC}_{95 \%} 13,9 ; 19,2\right]\right)\right)$ e Aracaju $\left(15,4 \%\right.$ [IC $_{95 \%}$ $13,02 ; 18,1])$ destacaram-se pelas maiores prevalências de simultaneidade de DCNTs, quando comparadas às demais capitais (Figura 2).

Em idosos, as cidades de Belém (34,1\% [ IC $_{95 \%}$ $28,6 ; 40,1])$, São Luís $\left(34,6 \%\right.$ [IC $\left.\left._{95 \%} 28,4 ; 40,5\right]\right)$ e Palmas (35,4\% [ $\left.\left.\mathrm{IC}_{95 \%} 26,4 ; 45,5\right]\right)$ tiveram as menores prevalências de simultaneidade de DCNTs, ao passo que Manaus $\left(57,1 \% \%\right.$ [ $\left.\left.\mathrm{IC}_{95 \%} 40,7 ; 53,5\right]\right)$, Belo Horizonte $\left(56,7 \%\right.$ [IC $\left.\left.\left._{95 \%} 41,5 ; 52,0\right]\right)\right)$ e Aracaju $(36,5 \%$ $\left.\left[\mathrm{IC}_{95 \%} 40,1 ; 53,0\right]\right)$ retrataram as maiores prevalências (Figura 3).

\section{Discussão}

Nas capitais brasileiras, observou-se uma importante diferença na prevalência de simultaneidade de DCNTs quando comparados adultos (13,7\%) e idosos (42,9\%). Pertencer à faixa etária de 40 a 59 anos, ser do sexo feminino, encontrar-se no estado civil-vivendo com companheiro, apresentar menor escolaridade e ser residente da região Sudeste do país contribuíram com maior presença proporcional no quadro de saúde de desfecho. Outrossim, as ocorrências de simultaneidade de doenças foram similares nas capitais brasileiras investigadas.

A prevalência de simultaneidade de DCNTs entre adultos identificada no presente estudo foi semelhante às apresentadas nos resultados de estudos conduzidos em países de alta renda, como o Canadá $e^{6}$ a Inglaterra. ${ }^{15}$ Idosos referiram valores mais elevados do que a estimativa de $30 \%$ prevista para países de alta renda ${ }^{16}$ resultado já encontrado nas prevalências de DCNTs separadamente, também mais prevalentes na população de países de baixa e média renda se comparados com aqueles de alta renda. ${ }^{17}$

Estudos realizados na Austrália, no ano de 2005, ${ }^{18}$ e no Canadá, ${ }^{19}$ entre 2003 e 2009 , encontraram prevalências de simultaneidade de doenças em adultos semelhantes às observadas nesta pesquisa, com valores de $32,6 \%$ e $28,2 \%$ respectivamente. Nos 
Tabela 1 - Descrição das características sociodemográficas, estratificada pela existência de simultaneidade de doenças crônicas não transmissíveis (DCNTs) (n=47.218), Brasil, 2013

\begin{tabular}{|c|c|c|c|c|c|c|c|c|}
\hline \multirow{2}{*}{$\begin{array}{l}\text { Características } \\
\text { sociodemográficas }\end{array}$} & \multicolumn{4}{|c|}{$\begin{array}{l}\text { Sem simultaneidade ( } 0 \text { a } 1 \text { doença) } \\
(n=36.717)\end{array}$} & \multicolumn{4}{|c|}{$\begin{array}{l}\text { Com simultaneidade ( } 2 \text { a } 4 \text { doenças) } \\
\text { ( } n=10.501)\end{array}$} \\
\hline & n & $\%^{a}$ & IC $_{95 \%}{ }^{\text {b }}$ & $\begin{array}{c}\% \\
\text { perda }\end{array}$ & n & $\%$ & IC $_{95 \%}{ }^{\text {b }}$ & $\begin{array}{c}\% \\
\text { perda }\end{array}$ \\
\hline \multicolumn{9}{|l|}{ Sexo } \\
\hline Masculino & 15.281 & 49,3 & $(48,3 ; 50,3)$ & & 3.801 & 44,0 & $(41,9 ; 46,2)$ & \\
\hline Feminino & 21.436 & 50,7 & $(497 ; 51,7)$ & & 6.700 & 56,0 & $(53,8 ; 58,1)$ & \\
\hline \multicolumn{9}{|l|}{ Idade (em anos) } \\
\hline $18-29$ & 8.666 & 33,7 & $(32,8 ; 34,8)$ & & 297 & 3,8 & $(3,2 ; 4,6)$ & \\
\hline $30-39$ & 7.071 & 24,5 & $(23,6 ; 25,4)$ & & 783 & 13,0 & $(11,5 ; 14,7)$ & \\
\hline $40-49$ & 7.300 & 18,7 & $(18,0 ; 19,5)$ & & 1.572 & 19,5 & $(17,8 ; 21,3)$ & \\
\hline $50-59$ & 6.276 & 12,3 & $(11,7 ; 12,9)$ & & 2.702 & 27,2 & $(25,3 ; 29,1)$ & \\
\hline $60-69$ & 4.158 & 6,3 & $(5,9 ; 6,7)$ & & 2.905 & 21,0 & $(19,4 ; 22,6)$ & \\
\hline $70-79$ & 2.360 & 3,2 & $(2,9 ; 3,5)$ & & 1.707 & 11,8 & $(10,7 ; 13,0)$ & \\
\hline $80-79$ & 801 & 1,2 & $(1,1 ; 1,4)$ & & 510 & 3,5 & $(2,9 ; 4,3)$ & \\
\hline$\geq 90$ & 85 & 0,05 & $(0,01 ; 0,1)$ & & 25 & 0,2 & $(0,1 ; 0,3)$ & \\
\hline Situação conjugal & & & & 0,9 & & & & 0,9 \\
\hline Sem companheiro(a) & 18.149 & 52,9 & $(51,9 ; 53,9)$ & & 4.424 & 35.6 & $(33,7 ; 37,6)$ & \\
\hline Com compheiro(a) & 18.256 & 47,1 & $(46,1 ; 48,1)$ & & 5.986 & 64,4 & $(62,4 ; 66,3)$ & \\
\hline Raça/cor da pele & & & & 11,0 & & & & 14,5 \\
\hline Branca & 15.600 & 47,3 & $(46,3 ; 48,4)$ & & 4.614 & 50,3 & $(48,0 ; 52,5)$ & \\
\hline Preta/parda & 17.069 & 52,7 & $(51,6 ; 53,8)$ & & 4.367 & 49,7 & $(47,5 ; 52,0)$ & \\
\hline Escolaridade (em anc & & & & 0,8 & & & & 1,0 \\
\hline $0-8$ & 7.518 & 29,3 & $(28,36 ; 30,3)$ & & 4.102 & 56,3 & $(54,3 ; 58,3)$ & \\
\hline $9-11$ & 14.211 & 40,5 & $(39,5 ; 41,5)$ & & 3.437 & 26,3 & $(24,8 ; 27,9)$ & \\
\hline$\geq 12$ & 14.707 & 30,2 & $(29,3 ; 31,1)$ & & 2.854 & 17,4 & $(16,0 ; 18,8)$ & \\
\hline \multicolumn{9}{|c|}{ Macroregiões nacionais } \\
\hline Centro-0este & 5.459 & 12,0 & $(11,6 ; 12,5)$ & & 1.623 & 10,1 & $(9,4 ; 10,9)$ & \\
\hline Nordeste & 11.865 & 24,8 & $(24,1 ; 25,5)$ & & 3.534 & 22,9 & $(21,6 ; 24,2)$ & \\
\hline Norte & 9.933 & 10,2 & $(9,8 ; 10,6)$ & & 2.381 & 8,5 & $(7,8 ; 9,2)$ & \\
\hline Sudeste & 5.367 & 44,3 & $(43,2 ; 45,4)$ & & 1.694 & 50,6 & $(48,5 ; 52,6)$ & \\
\hline Sul & 4.093 & 8,7 & $(8,3 ; 9,1)$ & & 1.269 & 7,9 & $(7,3 ; 8,7)$ & \\
\hline
\end{tabular}

a) Percentual na amostra ponderada.

b) Intervalo de confiança de $95 \%$ na amostra ponderada. 


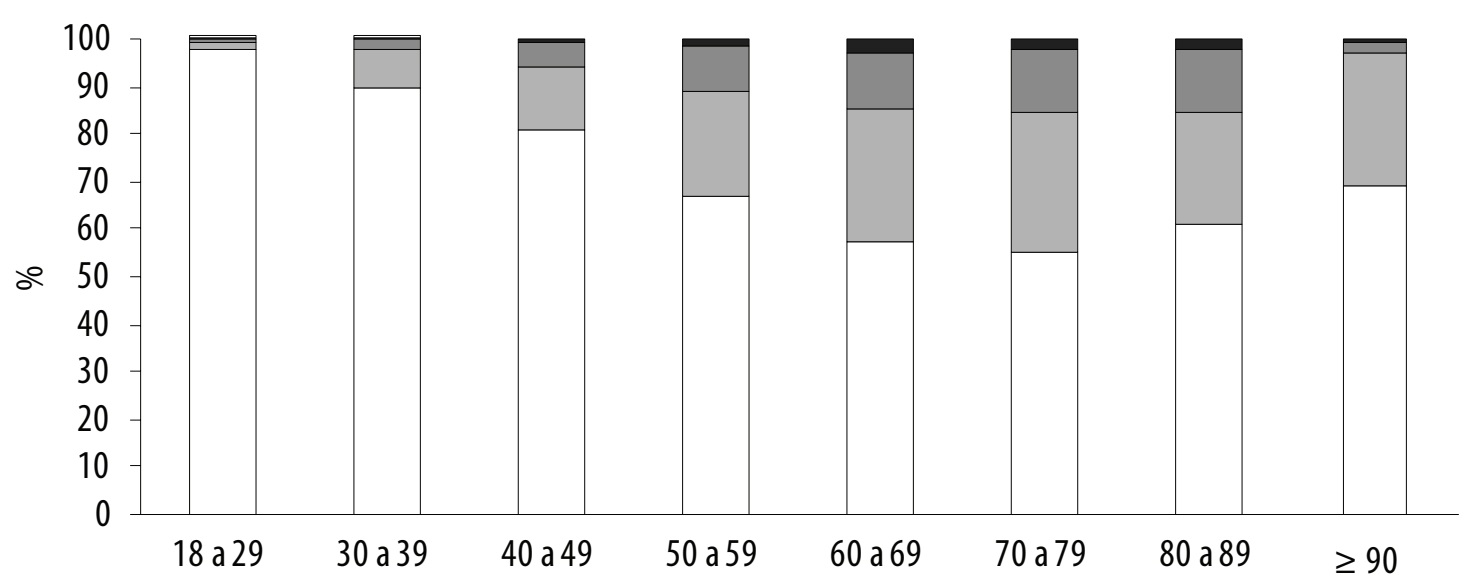

a) Valores ponderados

Figura 1 - Prevalência de doenças crônicas não transmissíveis (DCNTs) acumuladas, conforme faixas etárias $(\mathrm{n}=52.929)$, Brasil, 2013

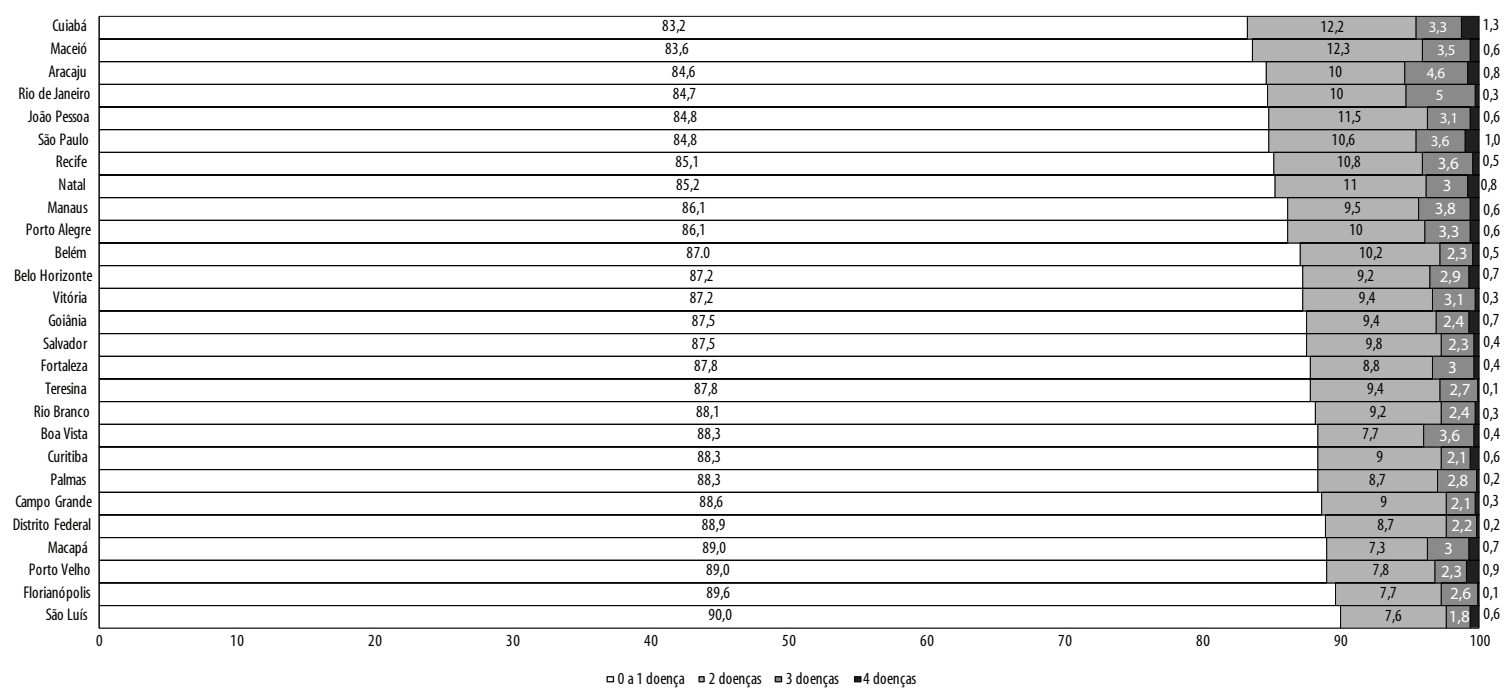

a) Valores ponderados.

Figura 2 - Prevalência do número de diagnósticos de doenças crônicas não transmissíveis (DCNTs) da amostra em adultos, estratificada por capitais das Unidades da Federação ( $n=37.947)$, Brasil, 2013 


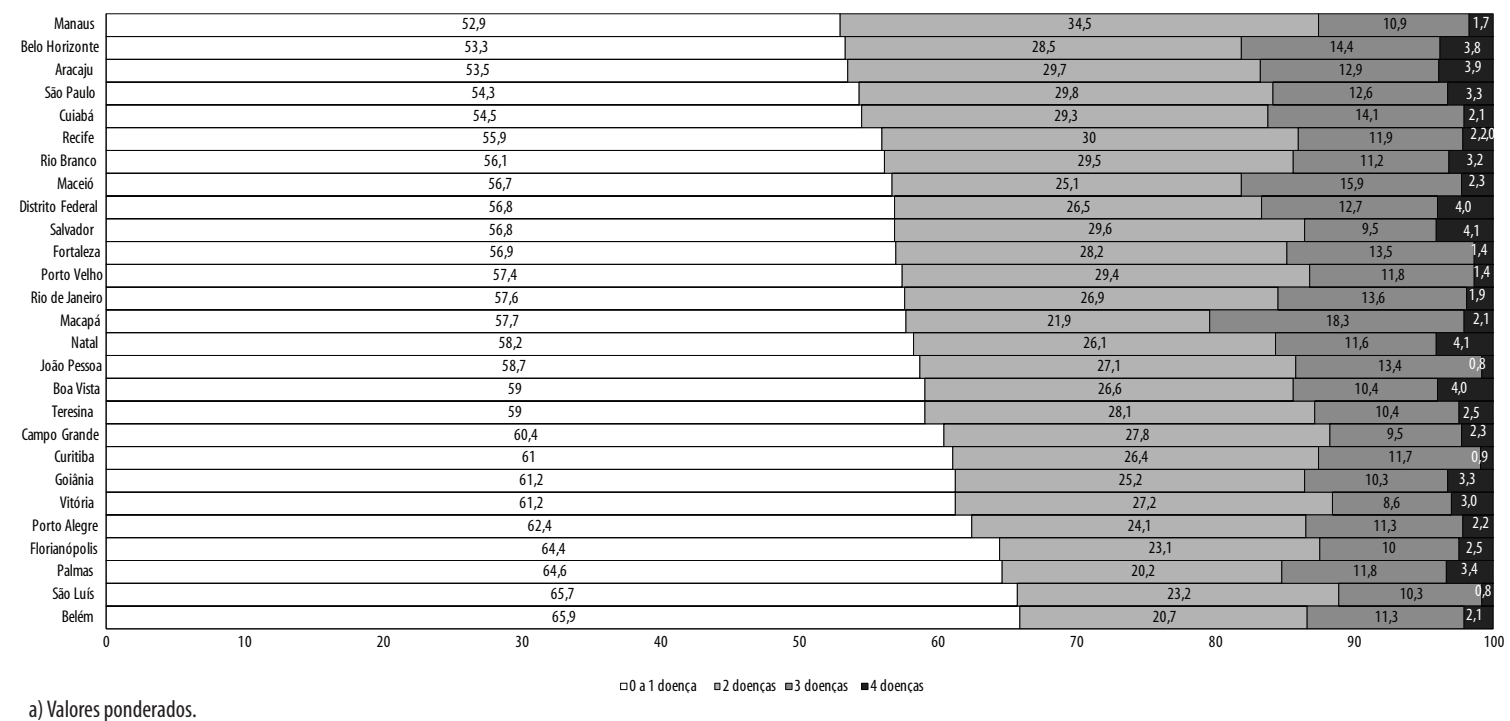

\section{Figura 3 - Prevalênciaa do número de diagnósticos de doenças crônicas não transmissíveis (DCNT) da amostra em idosos, estratificada por capitais das Unidades da Federação ( $n=14.982)$, Brasil, 2013}

dois países citados, a variação da simultaneidade de DCNTs também apresentou valores próximos aos do presente estudo, de $8,2 \%$ a $14,7 \%$ para duas, $1,9 \%$ a 3,9\% para três e $0,5 \%$ a $1,1 \%$ para quatro ou mais doenças, respectivamente. ${ }^{18,19}$ Sobre esse dois países, outros dois estudos também identificam um aumento proporcional dessas prevalências com 0 avançar da idade. ${ }^{6,15}$ Entretanto, um levantamento realizado na Índia vincula o fato de ter DCNT a maior escolaridade e renda, possivelmente atribuído à maior oportunidade de acesso a serviços de saúde. ${ }^{20}$

o Brasil apresenta semelhanças com países de maior renda, quanto à simultaneidade de doenças. É possivel que tais semelhanças se devam à redução de iniquidades em saúde pelas ações do Sistema Único de Saúde do Brasil, visto que o SUS oportuniza o diagnóstico e 0 acesso aos serviços de saúde para portadores de DCNT. ${ }^{21}$ Entretanto, estudos da Pesquisa Nacional de Saúde (PNS) identificaram que, embora os portadores de DCNT tenham mais acesso aos serviços de saúde do que os não portadores dessas doenças, ${ }^{21}$ referir menor escolaridade leva a maior incapacidade e, consequentemente, menor expectativa de vida saudável. ${ }^{22}$

Entre os idosos, foi possível identificar diferença entre os resultados do presente estudo e o realizado no Canadá, onde a prevalência de simultaneidade de doenças foi de $76,8 \%$ e a quantidade de duas, três e quatro doenças foi de $21,5 \%, 20,6 \%$ e
$34,7 \%$ respectivamente. ${ }^{22}$ Tal discrepância pode ser atribuída à quantidade de doenças investigadas nos estudos e, dado o fato de Brasil e Canadá possuírem serviços de saúde de provimento público, à mediação de diferentes determinantes sociais. ${ }^{22}$ Considerando-se que a simultaneidade de doenças resulta em internações hospitalares 5,6 vezes maiores na população idosa, ${ }^{8}$ aqueles com melhor apoio à educação, caso de países de alta renda, possuem maior controle das doenças existentes e, consequentemente, melhor qualidade de sobrevida, uma possível justificativa para a diferença observada.

Sabe-se, também, que a simultaneidade de doenças se reflete em um aumento de 5,5 vezes nos gastos de serviço de saúde, ${ }^{8}$ e que a falta de recursos para tal acompanhamento pode não permitir 0 tratamento adequado, aumentando o risco de mortalidade precoce. Acredita-se que aqueles que apresentam simultaneidade de doenças e maior sobrevida são os de maior escolaridade, enquanto o menor tempo de estudo contribui para uma mortalidade mais precoce. ${ }^{10}$

0 decréscimo de simultaneidade de doenças observado nas últimas décadas de vida, em ambos os sexos, não deve ser interpretado como um fator de proteção pela idade mais avançada, uma vez que essa simultaneidade se deve, principalmente, ao fato de pessoas com multimortalidade apresentarem maior chance de mortalidade em idades mais precoces. ${ }^{13} 0$ estudo de 
Britt et al., ${ }^{18}$ realizado com cidadãos australianos, identificou na quantidade de doenças em simultaneidade uma tendência de aumento conforme a década de vida em adultos, principalmente concentrada entre os $40 \mathrm{e}$ os 60 anos. Após esse aparecimento expressivo de mais doenças simultâneas, observa-se, nos anos seguintes, um decréscimo dessa quantidade na simultaneidade. Esse comportamento da simultaneidade pode ser atribuído ao viés de sobrevivência, segundo o qual indivíduos com mais de uma DCNT têm maior risco de mortalidade em razão dos agravos que as doenças, instaladas individualmente, geram no organismo com o passar do tempo. ${ }^{10}$ Ou seja, para um adulto, a partir de um primeiro diagnóstico, a exposição à simultaneidade será maior e, consequentemente, aumentará o risco para um segundo, um terceiro diagnóstico... aumentando a possibilidade de uma mortalidade precoce. Assim, idosos sem a simultaneidade de múltiplas DCNTs serão aqueles representados nas investigações do perfil de faixas etárias mais avançadas. As estratégias adotadas no cuidado primário à saúde são determinantes para o controle de risco de mortalidade, e devem ser desenvolvidas após a identificação de um perfil de saúde - ou falta de saúde - que permita identificar um organismo passível de maior exposição. ${ }^{17} \mathrm{O}$ avanço da idade, tanto para homens quanto para mulheres, representa estar mais exposto à simultaneidade de DCNTs desde a idade adulta. ${ }^{13}$ Homens e mulheres possuem um comportamento semelhante nas primeiras décadas de vida, quando ocorre um aumento dessa exposição às DCNTs, até a entrada na faixa etária idosa. Na fase adulta, existe a maior ocorrência de simultaneidade de doenças em homens, ao passo que, entre os idosos, as mulheres são mais atingidas, sugerindo que ainda não foi estabelecido em qual sexo a simultaneidade de doenças permanece mais evidente, independentemente da idade: ora os homens possuem maiores prevalências de simultaneidade de doenças, ora as mulheres. Tal variabilidade, encontrada na literatura, pode ser justificada por características das doenças investigadas, as quais possuem comportamento distinto em homens e mulheres, ao longo do ciclo vital. ${ }^{13} 0$ ápice encontrado para ambos os sexos coincide com a entrada na faixa etária idosa, época de vida cujas alterações morfofuncionais, comportamentais e emocionais são mais acentuadas. ${ }^{22}$
Aqueles com companheiros apresentaram maior prevalência de simultaneidade de doenças. Investigações sobre a existência da simultaneidade, sem atribuir diferença ao acúmulo de doenças, encontraram resultados semelhantes em idosos. ${ }^{23} \mathrm{Tal}$ fato pode ser justificado pela transição de situação conjugal naqueles que passam a viver com companheiros e adotar comportamentos não saudáveis, tais como redução dos níveis de atividade física, piora no padrão alimentar e de sono. ${ }^{24}$

Na população das capitais brasileiras, a raça/cor da pele não apresentou prevalências distintas entre duas categorias. Embora considerada uma variável de investigação, ${ }^{25} 0$ fato de não haver um ajuste de outros fatores para indicar associação pode levantar a discussão sobre a complexidade de diagnósticos em diferentes raças/cores da pele, mais além de aspectos socioculturais, tais como escolaridade e renda. Em análises estatísticas, a raça/cor da pele pode ser considerada como variável de confundimento residual de fatores socioeconômicos, os quais podem refletir um processo discriminatório ao longo da história enquanto forem identificados como risco a determinada condição de saúde. ${ }^{26}$

A relação entre a renda e a saúde está estabelecida na literatura: uma melhor posição social reflete-se em uma saúde melhor. ${ }^{27} 0$ nível educacional, por sua vez, é adotado como um importante marcador de desigualdade social, e a associação com a simultaneidade de doenças já foi encontrada naqueles com menor escolarização. ${ }^{8}$

Sobre as informações demográficas, o presente estudo apresentou a prevalência de 18,2\% de simultaneidade de DCNTs no total da amostra, valor inferior ao de $23,6 \%$ encontrado por um inquérito nacional realizado no mesmo ano, em todo o território brasileiro. A considerar 0 indicador de urbanização, sabe-se que pessoas residentes em zona urbana apresentam menor exposição à simultaneidade de doenças quando comparados àquelas residentes em zona urbana/rural. ${ }^{28,29}$

Quanto às macrorregiões nacionais, o Sudeste apresentou maior prevalência, possivelmente atribuída a aspectos como a densidade demográfica. ${ }^{27}$ Houve uma variação relativamente baixa, de pontos percentuais, nas prevalências de simultaneidade de DCNTs em adultos $(6,8 \%)$ e idosos (13\%), entre as diferentes capitais brasileiras. Tal fato pode ser atribuído aos valores semelhantes do índice de desenvolvimento humano (IDH) entre essas capitais, mesmo que elas disponham de orçamentos desi- 
guais, por exemplo, no que diz respeito aos gastos em saúde. ${ }^{29}$ Cumpre lembrar que o IDH é medido por uma combinação de fatores, entre os quais se encontram as condições de saúde, educação e saneamento.

Por fim, as cidades de Manaus, Belo Horizonte, Aracaju, Cuiabá e Maceió referiram as maiores prevalências de simultaneidade de DCNTs. Tal achado pode ser reflexo de alguns determinantes sociais, ${ }^{13}$ como a transição demográfica, o processo de urbanização, o crescimento econômico e social, ademais da diferença dos investimentos em serviços de saúde. No mesmo ano de realização do inquérito, o orçamento público em saúde dos estados a que as referidas capitais correspondem e o custo de saúde por habitante, ${ }^{29}$ com exceção de Amazonas e Sergipe, situava-se no tercil inferior do cenário nacional. 0 presente estudo se destaca pela investigação de caráter descritivo do perfil de adultos e idosos brasileiros com simultaneidade de DCNTs. A ocorrência de simultaneidade de doenças ao longo do ciclo de vida e seu comportamento segundo diferentes estratos de idade permitiu descrever, detalhadamente, o perfil das DCNTs no país, o que pode favorecer futuras medidas de caráter preventivo e de promoção da saúde na Atenção Primária oferecidas pelo SUS.

As capitais das UFs de todas as macrorregiões foram analisadas separadamente, o que permite compreender como a multimorbidade se distribui no Brasil. A confiabilidade da metodologia aplicada, por sua vez, possibilita a interpretação dos dados para a população geral em razão do poder amostral expressivo, e mais além, insere o presente estudo no cenário epidemiológico internacional como uma investigação cujos dados representam a condição de um país latino-americano de média renda.

Entretanto, algumas limitações devem ser pontuadas, como a medida de autorrelado de indicadores sociodemográficos e dos diagnósticos de doença, usualmente aplicada em estudos transversais, em que os valores podem ser superestimados ou subestimados segundo o conhecimento do entrevistado. o Brasil conta com diversos programas de Estado dirigidos à Saúde Pública, responsáveis por definir e informar as ações dos profissionais envolvidos na Atenção Primária, como a Estratégia Saúde da Família. ${ }^{30}$ Outrossim, esta análise considerou um conjunto limitado de doenças, sem a inclusão de outras enfermidades cardiovasculares, osteomusculares, de ordem mental e respiratórias, por exemplo. Contudo, os diagnósticos investigados se destacam no cenário nacional, reconhecidos nas diretrizes da área da Saúde como importantes indicadores de risco cardiovascular. ${ }^{12}$ Por fim, mesmo que se trate de uma amostra populacional do Brasil, a cobertura do inquérito utilizado considerou apenas residentes nas capitais dos estados, atendidos pelos serviços de telefonia fixa, o que restringe a extrapolação dos achados. 0 Vigitel realiza uma ponderação dos dados, destinada a reduzir os efeitos desse viés. ${ }^{14}$

Conclui-se que a simultaneidade de DCNTs em todas as capitais brasileiras condiz com o cenário epidemiológico mundial. 0 período de transição da faixa etária adulta para a idosa representou a fase mais crítica para a ocorrência de simultaneidade dessas doenças no ciclo vital considerado, tanto para homens quanto para mulheres. Como futuras aplicações práticas, sugere-se o acompanhamento das características e do estilo de vida ao longo de toda a fase adulta, com o objetivo de prevenir ou minimizar as complicações esperadas para a faixa etária idosa em pessoas com hábitos não saudáveis. Também se aponta a necessidade da orientação dos brasileiros e brasileiras com diagnóstico de alguma doença crônica não transmissível, com recomendações de mudança de comportamento e adoção de um estilo de vida mais saudável, concomitantemente aos tratamentos medicamentosos prescritos.

\section{Contribuição dos autores}

Christofoletti M, Del Duca GF e Malta DC contribuíram na concepção e delineamento do estudo. Christofoletti M realizou a análise e interpretação dos dados. Christofoletti M, Del Duca GF, Gerage AM e Malta DC contribuíram na redação, revisão crítica relevante do conteúdo intelectual e aprovação da versão final do manuscrito. Os autores declaram-se responsáveis por todos os aspectos do trabalho, no sentido de garantir que as questões relacionadas à exatidão ou à integridade de qualquer parte do manuscrito sejam devidamente investigadas e resolvidas. 


\section{Referências}

1. World Health Organization. Noncommunicable diseases progress Monitor [Internet]. Geneva: World Health Organization; 2017 [cited 2019 Sep 24]. 231 p. Available from: https://www.who.int/nmh/ publications/ncd-progress-monitor-2017/en/

2. Schramm JMA, Oliveira AF, Leite IC, Valente JG, Gadelha AMJ, Portela MC, et al. Transição epidemiológica e o estudo de carga de doença no Brasil. Ciênc Saúde Coletiva [Internet]. 2004 out-dez [citado 2019 set 24];9(1):897-08. Disponível em: http://www.scielo.br/pdf/csc/v9n4/a11v9n4.pdf. doi: 10.1590/\$1413-81232004000400011

3. Ministério da Saúde (BR). Secretaria de Atenção à Saúde. Departamento de Atenção Básica. Diretrizes para o cuidado das pessoas com doenças crônicas nas redes de atenção à saúde e nas linhas de cuidado prioritárias [Internet]. Brasília: Ministério da Saúde; 2013. 28 p. Disponível em: http://bvsms.saude. gov.br/bvs/publicacoes/diretrizes\%20_cuidado_ pessoas\%20_doencas_cronicas.pdf

4. Duncan BB, Chor D, Aquino EML, Bensenor IM, Mill JG, Schmidt MI, et al. Doenças crônicas não transmissíveis no Brasil: prioridade para enfrentamento e investigação. Rev Saúde Pública [Internet]. 2012 dez [citado 2019 set 24];46(1):126-34. Disponível em: http://www. scielo.br/pdf/rsp/v46s1/17.pdf. doi: 10.1590/S003489102012000700017

5. Ward BW, Schiller JS, Goodman RA. Multiple chronic conditions among US adults: a 2012 update. Prev Chronic Dis [Internet]. 2014 Apr [cited 2019 Sep 24];11(1):E62. Available from: https://www.ncbi.nlm. nih.gov/pmc/articles/PMC3992293/

6. Roberts KC, Rao DP, Bennett TL, Loukine L, Jayaraman GC. Prevalence and patterns of chronic disease multimorbidity and associated determinants in Canada. Health Promot Chronic Dis Prev Can [Internet]. 2015 Aug;35(6):87-94. Available from: https://www.ncbi.nlm.nih.gov/pmc/articles/ PMC4910465/

7. Birriel JT, Uchino R, Barry N, Butryn T, Sabol DM, Valenza PL, et al. Adverse drug reactions in the era of multi-morbidity and polypharmacy. J Basic Clin Pharm [Internet]. 2015 Sep-Nov [cited 2019 Sep 24];6(4):122-3. Available from: https://www. ncbi.nlm.nih.gov/pmc/articles/PMC4660485/. doi: 10.4103/0976-0105.168052
8. Bähler C, Huber CA, Brüngger B, Reich 0. Multimorbidity, health care utilization and costs in an elderly community-dwelling population: a claims data based observational study. BMC Health Ser Res [Internet]. 2015 Jan ]cited 2019 Sep 24];15(1):23 Available from: https://www.ncbi.nlm.nih.gov/pmc/ articles/PMC4307623/. doi: 10.1186/s12913-0150698-2

9. Wittenberg R. The challenge of measuring multimorbidity and its costs. Isr J Health Policy Res [Internet]. 2015 Jan [cited 2019 Sep 24];4(1)1. Available from: https://www.ncbi.nlm.nih.gov/pmc/ articles/PMC4422152/. doi: 10.1186/2045-4015-4-1

10. Nunes BP, Thumé E, Facchini LA. Multimorbidity in older adults: magnitude and challenges for the Brazilian health system. BMC Public Health [Internet]. 2015 Nov [cited 2019 Sep 24];15(1):1172. Available from: https://www.ncbi.nlm.nih.gov/pmc/articles/ PMC4658761/. doi: 10.1186/s12889-015-2505-8

11. Amaral TLM, Amaral CDA, Lima NSD, Herculano PV, Prado PRD, Monteiro GTR. Multimorbidade, depressão e qualidade de vida em idosos atendidos pela Estratégia de Saúde da Família em Senador Guiomard, Acre, Brasil. Ciênc Saúde Coletiva [Internet]. 2018 set;23(9):3077-84. Disponível em: http://www.scielo.br/pdf/csc/v23n9/14138123-csc-23-09-3077.pdf. doi: 10.1590/141381232018239.22532016

12. Violan C, Foguet-Boreu Q, Flores-Mateo G, Salisbury C, Blom J, Freitag M, et al. Prevalence, determinants and patterns of multimorbidity in primary care: a systematic review of observational studies. PLoS One [Internet]. 2014 Jul [cited 2019 Sep 24];9(7):e102149. Available from: https://www. ncbi.nlm.nih.gov/pmc/articles/PMC4105594/. doi: 10.1371/journal.pone.0102149

13. Ministério da Saúde (BR). Secretaria de Vigilância em Saúde. Departamento de Análise de Situação de Saúde. Plano de ações estratégicas para o enfrentamento das doenças crônicas não transmissíveis (DCNT) no Brasil 2011-2022 [Internet]. Brasília: Ministério da Saúde; 2011 [citado 2019 set 24]. 160 p. Disponível em: http:// bvsms.saude.gov.br/bvs/publicacoes/plano_acoes_ enfrent_dcnt_2011.pdf

14. Ministério da Saúde (BR). Secretaria de Vigilância em Saúde. Departamento de Doenças e Agravos não Transmissíveis e Promoção da Saúde. Vigilância de fatores de risco e proteção para doenças crônicas por 
inquérito telefônico, Vigitel 2014 [Internet]. Brasília: Ministério da Saúde; 2015 [citado 2019 set 24]. 152 p. Disponível em: http://bvsms.saude.gov.br/bvs/ publicacoes/vigitel_brasil_2014.pdf

15. Salisbury C, Johnson L, Purdy S, Valderas J, Montgomery A. Epidemiology and impact of multimorbidity in primary care: a retrospective cohort. Br J Gen Pract [Internet]. 2011 Jan [cited 2019 Sep 24];61(582):e12-21. Available from: https://bjgp.org/content/61/582/e12.long. doi: 10.3399/bjgp11X548929

16. Tacken MA, Opstelten W, Vossen I, Smeele IJ, Calsbeek $\mathrm{H}$, Jacobs JE, et al. Increased multimorbidity in patients in general practice in the period 20032009. Ned Tijdschr Geneeskd [Internet]. 2011 [cited 2019 Sep 24];155(26):A3109. Available from: https://www.ntvg.nl/artikelen/toenamemultimorbiditeit-bij-patiënten-de-huisartsenpraktijkde-periode-2003-2009.

17. Abegunde DO, Mathers CD, Adam T, Ortegon M, Strong K. The burden and costs of chronic diseases in low-income and middle-income countries. Lancet [Internet]. 2007 Dec [cited 2019 Sep 24];370(9603):1929-38. Available from: https:// www.thelancet.com/journals/lancet/article/PIIS01406736(07)61696-1/fulltext. doi: 10.1016/S01406736(07)61696-1

18. Britt HC, Harrison CM, Miller GC, Knox SA. Prevalence and patterns of multimorbidity in Australia. Med J Aust [Internet]. 2008 Jul [cited 2019 Sep 24];189(2):72-7. Available from: https://onlinelibrary. wiley.com/doi/abs/10.5694/j.1326-5377.2008. tb01919.x?sid=nlm\%3Apubmed. doi: 10.5694/ j.1326-5377.2008.tb01919.x

19. Pefoyo AJK, Bronskill SE, Gruneir A, Calzavara A, Thavorn K, Petrosyan Y, et al. The increasing burden and complexity of multimorbidity. BMC Public Health [Internet]. 2015 [cited 2019 Sep 24];15(1). Available from: https://bmcpublichealth.biomedcentral.com/ articles/10.1186/s12889-015-1733-2

20. Vellakkal S, Subramanian SV, Millett C, Basu S, Stuckler D, Ebrahim S. Socioeconomic inequalities in noncommunicable diseases prevalence in India: disparities between self-reported diagnoses and standardized measures. PLoS One [Internet]. 2013 [cited 2019 Sep 24];8(7):e68219. Available from: doi:

21. Menotti A, Mulder I, Nissinen A, Giampaoli S, Feskens EJM, Kromhout D. Prevalence of morbidity and multimorbidity in elderly male populations and their impact on 10-year all-cause mortality: the FINE study (Finland, Italy, Netherlands, Elderly). J Clin Epidemiol. 2001 Jul;54(1):680-6.

22. Del Duca GF, Silva MC, Hallal PC. Incapacidade funcional para atividades básicas e instrumentais da vida diária em idosos. Rev Saúde Pública [Internet]. 2009 out [citado 2019 set 24];43(5):796805. Disponível em: http://www.scielo.br/ pdf/rsp/v43n5/653.pdf. doi: 10.1590/S003489102009005000057

23. Banjare P, Pradhan J. Socio-economic inequalities in the prevalence of multi-morbidity among the rural elderly in Bargarh District of Odisha (India). PloS One [Internet]. 2014 Jun [cited 2019 Sep 24];9(6):e97832. Available from: https:// journals.plos.org/plosone/article?id=10.1371/ journal.pone.0097832. doi: 10.1371/journal. pone. 0097832

24. Robles TF. Marital quality and health: implications for marriage in the 21(st) century. Curr Dir Psychol Sci [Internet]. 2014 Dec [cited 2019 Sep 24];23(6):427-32. Available from: https://www. ncbi.nlm.nih.gov/pmc/articles/PMC4275835/. doi: 10.1177/0963721414549043

25. St Sauver JL, Boyd CM, Grossardt BR, Bobo WV, Finney Rutten LJ, Roger VL, et al. Risk of developing multimorbidity across all ages in an historical cohort study: differences by sex and ethnicity. BMJ Open [Internet]. 2015 Feb [cited 2019 Sep 24];5(2):e006413. Available from: https://bmjopen.bmj.com/content/5/2/e006413. long. doi: 10.1136/bmjopen-2014-006413

26. Kabad JF, Bastos JL, Santos RV. Raça, cor e etnia em estudos epidemiológicos sobre populações brasileiras: revisão sistemática na base PubMed. Physis [Internet]. 2012 [citado 2019 set 24];22(3):895-918. Disponível em: http://www. scielo.br/pdf/physis/v22n3/04.pdf. doi: 10.1590/ S0103-73312012000300004

27. Bambra C, Gibson M, Sowden A, Wright K, Whitehead M, Petticrew M. Tackling the wider social determinants of health and health inequalities: evidence from systematic reviews. J Epidemiol Community Health [Internet]. 2010 [ cited 2019 Sep 24];64(4):284-91. Available from: https://jech.bmj.com/content/jech/64/4/284.full. pdf. doi: 10.1136/jech.2008.082743

28. Afshar S, Roderick PJ, Kowal P, Dimitrov BD, Hill AG. Multimorbidity and the inequalities of global 
ageing: a cross-sectional study of 28 countries using the World Health Surveys. BMC Public Health [Internet]. $2015 \mathrm{Aug}$ [cited 2019 Sep 24];15(1):776. Available from: https://www.ncbi. nlm.nih.gov/pmc/articles/PMC4534141/. doi: 10.1186/s12889-015-2008-7

29. Ministério da Saúde (BR). Secretaria Executiva. Datasus. Sistema de informações sobre orçamentos públicos em saúde indicadores estaduais (SIOPS) [Internet]. Brasília: Ministério da Saúde; 2013 [citado $2016 \mathrm{dez} 5$ ]. Disponível em: http://siopsasp.datasus.gov.br/cgi/tabcgi.exe?SIOPS/SerHist/ ESTADO/indicuf.def

\begin{abstract}
Objective: to describe the prevalence and sociodemographic profile of chronic noncommunicable disease (CNCD) simultaneity in adults and elderly people resident in Brazilian state capital cities. Methods: Chronic Noncommunicable Disease Risk and Protection Factor Surveillance System Survey 2013; simultaneity was considered to be two or more CNCDs (diabetes mellitus, dyslipidemia, hypertension, and obesity). Results: of the total 52,929 participants, $13.7 \%$ of adult participants and $42.9 \%$ of elderly participants had CNCD simultaneity; bypertension and diabetes mellitus simultaneity was greater in adults, while hypertension and obesity simultaneity was greater in the elderly; simultaneity was more prevalent in women, in those between 50 and 59 years old, with partners and up to eight years of schooling; the cities with the lowest and highest prevalence in adults were São Luis and Cuiaba, respectively, while in the elderly, the cities were Belém and Manaus, respectively. Conclusion: simultaneity was identified nationwide; prevention measures should be directed especially toward treatment of hypertension.

Keywords: Chronic Disease; Diagnosis-Related Groups; Socioeconomic Factors; Cross-Sectional Studies.
\end{abstract}

30. Malta DC, Santos MAS, Stopa SR, Vieira JEB, Melo EA, Reis C. A cobertura da estratégia de saúde da família (ESF) no Brasil, segundo a pesquisa nacional de saúde, 2013. Ciênc Saúde Coletiva [Internet]. 2016 fev [citado 2019 set 24];21(2):327-38. Disponível em: http://www.scielo.br/pdf/csc/v21n2/14138123-csc-21-02-0327.pdf. doi: 10.1590/141381232015212.23602015

\section{Resumen}

objetivo: describir la prevalencia y el perfil sociodemográfico de la simultaneidad de enfermedades crónicas no transmisibles (ECNT) en adultos y ancianos de las capitales brasileñas. Métodos: Sistema de Vigilancia de Factores de Riesgo y Protección para Enfermedades Crónicas no Transmisibles (Vigitel), realizado en 2013; la simultaneidad consideró dos o más ECNT (diabetes mellitus, dislipidemia, hipertensión y obesidad). Resultados: en 52.929 participantes, $13,7 \%$ de los adultos y el 42,9\% de los ancianos presentaron simultaneidad de ECNT; bubo mayor agrupamiento de hipertensión con diabetes mellitus en adultos e hipertensión con obesidad en ancianos; la mayor prevalencia fue en mujeres, con edad entre 50 y 59 años, con compañero y escolaridad de hasta ocho años; las ciudades con menory mayor prevalencia, respectivamente, en adultos fueron São Luísy Cuiabá, y en los ancianos, Belém y Manaus. Conclusión: la simultaneidad se identificó en el contexto nacional, y medidas de prevención deben ser dirigidas especialmente al tratamiento de la hipertensión.

Palabras clave: Enfermedad Crónica; Grupos Diagnósticos Relacionados; Factores Socioeconómicos; Estudios Transversales.

Recebido em 12/02/2019

Aprovado em 06/09/2019 